\title{
Antibiotic De-escalation Therapy in Neurosurgical Patients with Ventilator-Associated Pneumonia in Intensive Care Unit: A Retrospective Observational Study
}

\author{
Rahela Ambaras Khan, Zoriah Aziz* \\ Department of Pharmacy, Faculty of Medicine, University of Malaya- Kuala Lumpur, MALAYSIA.
}

\begin{abstract}
Context: Antibiotic de-escalation practice is gaining interest in the intensive care units (ICU). However, there is limited evidence to support this approach in neurosurgical patients with Ventilator-Associated Pneumonia (VAP) in ICU. Aims: This study examined the practice of antibiotic de-escalation in neurosurgical patients with VAP in ICU and its impact on mortality. Settings and Design: Retrospective cross-sectional study conducted in an ICU of a public hospital in Malaysia. Methods and Material: The electronic medical records of the patients diagnosed with VAP in the ICU were retrieved and relevant data was collected for analysis. Statistical analysis used: Mann-Whitney U-test and ChiSquare Test were used to compare the differences of continuous and categorical data while Logistic Regression and Cox Regression were used to estimate the influence of de-escalation on mortality. Results: Of the 125 VAP patients identified, only 53 (42.4\%) were neurosurgical patients. The mean age of these patients was $40.5 \pm 15.3$ years old. The patients mainly had late-onset VAP. The most common organisms identified included Pseudomonas aeruginosa (19.6\%) and Acinetobacter Multidrug-Resistant Organisms $(17.9 \%)$ while the most commonly used empirical antibiotic was Cefepime (32\%). The proportion of patients with antibiotic de-escalation (10 out of 53) was statistically lower than patients without antibiotic de-escalation (43 out of 53). There was no statistically significant difference in ICU-mortality between the de-escalation ( 2 out of 10) and non-deescalation groups (6 out of 43). Similarly, for the 28-day mortality and survival analysis, no significant difference was found between the two groups. Conclusions: Antibiotic de-escalation practice in neurosurgical patients with VAP in ICU was not associated with a deleterious effect on survival.
\end{abstract}

Key words: Antibiotic de-escalation, Ventilator-associated pneumonia, Neurosurgical, Critical care, Critically-ill patients.

Key Messages: Despite the potential benefit of antibiotic de-escalation, this practice remains low among the neurosurgical population with ventilator-associated pneumonia in the intensive care unit. As this practice was not associated with an excess in mortality, it should be cultivated among critically-ill neurosurgical patients in intensive care unit.

\section{INTRODUCTION}

Antibiotic de-escalation is a practice of starting with broad-spectrum empirical antibiotics and streamlining to the narrowerspectrum or fewer agents after thorough re-assessment of the patient's condition, inflammatory markers and microbiological data. ${ }^{1-4}$ Antibiotic de-escalation is a subset of antibiotic stewardship programme to promote the judicious use of antibiotics., The discriminate use of antibiotics is vital as it may assist long-term solution to curb the emergence of resistant organism ${ }^{7,8}$ In addition, with the lack of antibiotic development in the pipe-
Submission Date: 13-07-2016; Revision Date: 17-11-2016; Accepted Date: 22-11-2016 DOI: 10.5530/ijper.51.1.19 Correspondence: Zoriah Aziz, Department of Pharmacy, Faculty of Medicine, University of Malaya-Kuala Lumpur, Malaysia.

Phone no: +60379674909 E-mail: zoriah@um.edu.my

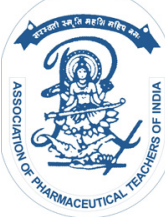

www.ijper.org 
line and the threat of global antimicrobial resistance, the antibiotic de-escalation is crucial to be implemented in the hospital setting.

The practice of antibiotic de-escalation is increasingly adopted in Intensive Care Units (ICU) ${ }^{9-13}$ as antibiotics are widely prescribed in this setting ${ }^{14,15}$ Among the infections in ICU, Ventilator-associated Pneumonia (VAP) is an important infection among critically ill patients and is a significant public health issue with increased morbidity and mortality. ${ }^{16}$ Several studies involving patients with VAP in ICU found that antibiotic de-escalation is a good strategy to conserve the effectiveness of broadspectrum antibiotics without compromising patients' outcome. ${ }^{10,12,17,18}$ However, there is limited evidence to support the de-escalation approach in specific neurosurgical patients with VAP in ICU. Neurosurgical patients are at risk of developing VAP and other nosocomial infections in ICU due to serious nature of their illnesses and the use of invasive devices. ${ }^{19,20}$ Hence, it is important to assess the practice of antibiotic de-escalation and how it is executed in neurosurgical patients. We designed a study to examine the practice of antibiotic de-escalation in neurosurgical patients diagnosed with VAP in ICU and the outcome on ICU-mortality and 28-day mortality.

\section{MATERIALS AND METHODS}

\section{Design and sample}

This was a retrospective cross-sectional study conducted in an ICU of a public hospital in Malaysia that serves as a tertiary referral centre for neurosurgery. Data for all VAP patients admitted to the ICU from January 2012 to December 2014 was searched from The Malaysian Registry of Intensive Care Database. The electronic medical records of the eligible patients were retrieved and recorded. The data collected included patients' demographic profiles, Simplified Acute Physiology Score (SAPS) II, Sequential Organ Failure Assessment (SOFA) Score, mechanical ventilation (MV) information, prescribed antibiotic, microbiological results, inflammatory markers, ICU - mortality and 28-d mortality. Paediatric patients, patients with missing data and patients who had died on the day of VAP diagnosis were excluded from analysis. The Ministry of Health Medical Research Ethics Committee approved this protocol waiving the need for informed consent since this was an observational study.

\section{Definition}

VAP was diagnosed when persistent, new pulmonary infiltrates appeared on chest radiographs more than 48 hours after endotracheal intubation, in conjunction with purulent respiratory secretions. At least one of the following criteria was also required: temperature greater than $38^{\circ} \mathrm{C}$ or less than $36^{\circ} \mathrm{C}$; blood leukocytes greater than $12,000 / \mu \mathrm{L}$ or less than $4000 / \mu \mathrm{L}$. Only VAP caused by bacterial pneumonia was selected. ICU-mortality was defined as a patient who died in ICU due to either the infection itself or complications related to the patients' concomitant diseases and 28-day mortality was defined as overall hospital mortality after 28 days discharge from ICU. SAPS II and SOFA Score were used to measure the severity of disease and to determine the extent of a patient's organ function respectively. Late-onset VAP was defined as VAP that occurs after 5 days of mechanical ventilation and infections are more likely to be caused by multidrug-resistant organisms ${ }^{[9]}$. Appropriate antibiotic at 24 hours (h) was defined as antibiotic which was initiated within $24 \mathrm{~h}$ of VAP diagnosis and was matched with culture and sensitivity results.

\section{Data analysis}

Data analysis was conducted using IBM $^{\circledR}$ SPSS $^{\circledR}$ Statistics Version 21. Mann-Whitney U-test was used to compare the differences of continuous data (not normally distributed) between two groups. For categorical data, Chi-Square or Fisher's Exact Test was used. Logistic regression and cox regression were used to estimate the influence of de-escalation therapy on mortality outcome. All tests of significance were two-tailed and $\mathrm{p}$ values of $<0.05$ were considered significant.

\section{RESULTS}

\section{Characteristic of neurosurgical patients}

Of the 125 of VAP patients identified, 53 (42.4\%) neurosurgical patients were included in the study (Table 1). The majority of the subjects were male with mean age of $40.5 \pm 15.3$ years old. The mean onset of VAP was $6.5 \pm 3.2$ days. This was considered as late-onset VAP. Patients were not on prolonged ventilation as the mean length of mechanical ventilation was $12.4 \pm 5.4$ ( $<21$ days). On admission, the mean SAPS II Score and SOFA Score were $42.2 \pm 14.20$ and $10.0 \pm 2.3$ respectively. Table 1 shows that there was no statistically significant difference between neurosurgical patients with and without de-escalation therapy for all characteristics.

\section{Microorganisms identified and antibiotic prescribed}

A causative pathogen was identified in 39 out of 53 patients with VAP. A total of 31 (79.5\%) samples were identified through tracheal aspirate (TA), 6 (15.4\%) samples through bronchoalveolar lavage (BAL) and 2 $(5.1 \%)$ from blood cultures. Table 2 shows 14 patients had unidentified organism (culture-negative) while the 
Table 1: Characteristic of neurosurgical patients with ventilator- associated pneumonia

\begin{tabular}{|c|c|c|}
\hline Characteristics & De-escalation $(\mathbf{n = 1 0 )}$ & Non-De-Escalation $(\mathbf{n}=\mathbf{4 3})$ \\
\hline Age, mean (SD), year & $45.1 \pm 11.0$ & $39.4 \pm 16.0$ \\
\hline Male, $\mathrm{n}(\%)$ & $5.0(50.0)$ & $37.0(86.0)$ \\
\hline VAP Onset, mean (SD), day & $7.4 \pm 3.1$ & $6.2 \pm 3.3$ \\
\hline Length of MV, mean (SD), day & $15.9 \pm 5.1$ & $11.5 \pm 5.2$ \\
\hline SAPS II Score, mean (SD) & $45.5 \pm 18.8$ & $41.4 \pm 13.9$ \\
\hline SOFA Score, mean (SD) & $9.9 \pm 2.1$ & $10.0 \pm 2.4$ \\
\hline Appropriate Antibiotic at 24 hours, $\mathrm{n}(\%)$ & $3.0(33.3)$ & $16.0(40.0)$ \\
\hline
\end{tabular}

\begin{tabular}{|c|c|c|c|}
\hline $\begin{array}{c}\text { Microorganisms \& } \\
\text { Antibiotics }\end{array}$ & Total; $\mathbf{n}(\%)$ & De-escalation; n (\%) & $\begin{array}{c}\text { Non-De-Escalation; } \\
\mathrm{n}(\%)\end{array}$ \\
\hline $\begin{array}{l}\text { Microorganisms identified: } \\
\text { Acinetobacter MDRO }\end{array}$ & $10(17.9)$ & $1(10.0)$ & $9(90.0)$ \\
\hline$P$. aeruginosa & $11(19.6)$ & $2(18.2)$ & $9(81.8)$ \\
\hline ESBL Organisms & $6(10.7)$ & $1(16.7)$ & $5(83.3)$ \\
\hline Enterobacter $s p$ & $2(3.6)$ & $2(100.0)$ & $0(0.0)$ \\
\hline Polymicrobial & $7(12.5)$ & $2(28.6)$ & $5(71.4)$ \\
\hline Others & $6(10.7)$ & $2(33.3)$ & $4(66.7)$ \\
\hline Culture-negative* & $14(25.0)$ & $0(0.0)$ & $14(100.0)$ \\
\hline \multicolumn{4}{|l|}{ Empirical Antibiotics Used: } \\
\hline Colistin & $6(11.3)$ & $3(50.0)$ & $3(50.0)$ \\
\hline Carbapenems & $9(17.0)$ & $1(11.1)$ & $8(88.9)$ \\
\hline Cefepime & $17(32.1)$ & $2(11.8)$ & $15(88.2)$ \\
\hline Piperacillin/Tazobactam & $5(9.4)$ & $1(20.0)$ & $4(80.0)$ \\
\hline Ampicllin/Sulbactam & $9(17.0)$ & $1(11.1)$ & $8(88.9)$ \\
\hline Cefazolin & $2(3.8)$ & $0(0.0)$ & $2(100.0)$ \\
\hline Comb. of broad spectrum antibiotics & $5(9.4)$ & $2(40.0)$ & $3(60.0)$ \\
\hline
\end{tabular}

[? Since $>1$ microorganisms possible for pneumonia, total count will exceed 53

* Statistically significant at $p<0.05$

two most frequently identified organisms were Pseudomonas aeruginosa and Acinetobacter Multidrug-Resistant Organisms (MDRO). Cefepime was the most commonly used empirical antibiotic followed by carbapenem and ampicillin/sulbactam (Table 2).

\section{De-escalation practice and the outcome on ICU- mortality and 28-d mortality}

The number of neurosurgical patients with antibiotic de-escalation was statistically lower than those without de-escalation therapy (10 versus 43). De-escalation to a narrow-spectrum antibiotic regimen occurred in eight patients while one or more antibiotics were withdrawn in two other patients.

ICU-mortality [Odd Ratio $=1.542 ; 95 \%$ CI $(0.262-$ 9.082)] was higher in de-escalation group compared to the non-de-escalation group, though, the difference was not statistically significant. Similarly, the 28 -day mortality in the de-escalation group [Odd Ratio $=1.619 ; 95 \%$ CI (0.347 - 7.546)] was not statistically significant compared to the non-de-escalation group.

As shown in Figure 1, there was no significant difference in survival between the two groups.

\section{DISCUSSIONS}

This study compared the practice of antibiotic de-escalation and its outcome among neurosurgical patients treated for VAP infection in ICU. In this study, empirical antibiotics were streamlined in about $20 \%$ of the cohort with VAP. This figure is lower than $25 \%$ to $35 \%$ found in the previous studies involving medical, surgical and trauma patients with VAP. ${ }^{10,12,21}$ The de-escalation rate was lower in our study compared to previous reports probably due to the types of patients included, as our study only included neurosurgical patients. 


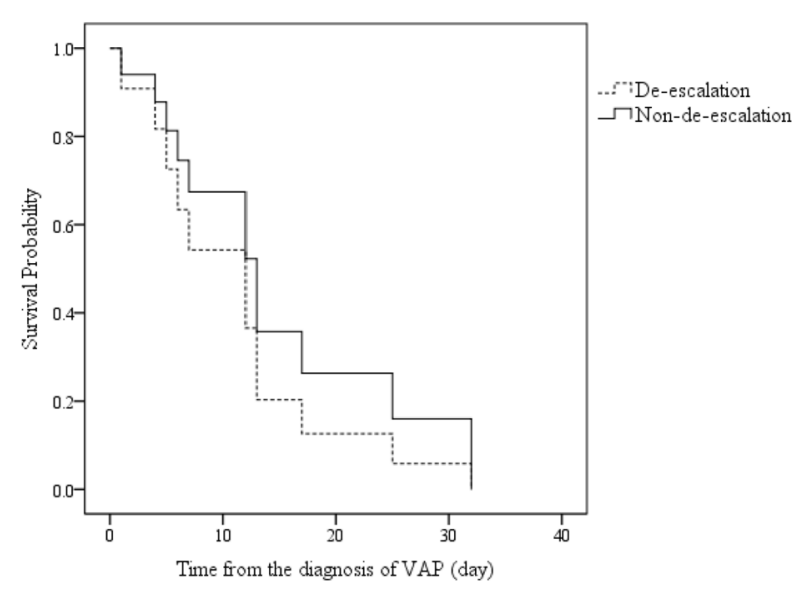

Figure 1: All-cause mortality for neurosurgical patients

Another factor that might influence the lower de-escalation rate in our study was the high proportion of unidentified organisms (culture-negative) from culture and sensitivity results. This might limits the opportunity for de-escalation. In the light of literature and guidelines, antibiotic de-escalation in culture-negative patients is not being adequately addressed. The decision about when and how to de-escalate is left to clinical judgment. In some circumstances, the culture-negative result does not correlate with the patient's severe condition and this contributed to the prolonged use of empirical antibiotics. Managing culture-negative result in septic patients is one of the known challenges of antibiotic de-escalation., ${ }^{3,13}$ Kollef et al (2005) recommended to discontinue antibiotic therapy within 72 hours or to completely withheld therapy in clinically suspected VAP patients with culture-negative BAL. ${ }^{22}$ Similarly, Alvarez-Lerma et al (2006) and Giantsou et al (2007) found better de-escalation strategy facilitated by BAL since it has higher specificity than TA cultures. ${ }^{11,23}$ In this study, BAL was used in only six patients. Hence, to improve the de-escalation strategy in the culture-negative result, BAL should be encouraged for confirmed case of VAP.

Antibiotic de-escalation practice should also be complemented with developing new diagnostic tools to determine causative organisms for VAP. With rapid diagnostic tools, clinicians can treat the infections appropriately and effectively without prolonging the duration of empirical antibiotic treatment. ${ }^{24}$

Eventhough the findings in our study were not statistically significant, neurosurgical patients without antibiotic de-escalation showed a better survival and lower risk of ICU-mortality and 28-d mortality. Nevertheless, this result could be influenced by a small number of sample size. Generally, most of the studies are in agreement with our findings, where no significant deleterious effect on survival rate was observed in those who underwent antibiotic de-escalation therapy. ${ }^{10,21}$ Indeed, Rello et al (2004) and Kollef (2006) found significantly lower mortality rate in de-escalation group with VAP. ${ }^{12,25}$

This study has several limitations. First, our study is a retrospective study involving only a single ICU setting with a small sample size. Second, our study involved closed ICU model in which only a few individuals have control of the antibiotic prescribing. Third, there is no standard protocol for antibiotic de-escalation. Further multicentre studies using standard antibiotic de-escalation protocol are needed to evaluate the impact of antibiotic de-escalation therapy in neurosurgical patients.

\section{CONCLUSIONS}

Despite the potential benefits of antibiotic de-escalation, our results showed low de-escalation rate in neurosurgical patients with VAP in ICU. Clinicians should be urged to identify strategies to promote the practice of antibiotic de-escalation in order to prevent the emergence of resistant organisms and to reduce resource utilisation. Thus, antibiotic de-escalation should be encouraged in neurosurgical patients with VAP in ICU as it was not associated with increased mortality.

\section{ACKNOWLEDGEMENT}

We would like to thank The Director General of Health, Ministry of Health Malaysia for the permission to publish this paper. We are indebted to Dr Shanthi Ratnam, Consultant Intensivist, Sungai Buloh Hospital, Dr Suresh Kumar, Consultant Infectious Disease, Sungai Buloh Hospital, Dr. Kalaiarasu Peariasamy, Senior Consultant and Head of Paediatric Dentistry, Sungai Buloh Hospital and SN Siti Salwa Mohd Latib for their support towards this research project. Special thanks also go to Faculty of Medicine, University of Malaya, Clinical Research Centre and Pharmacy Department of Sungai Buloh Hospital for their technical support.

\section{CONFLICT OF INTEREST}

No conflict of interest are declared.

\section{ABBREVIATIONS USED}

ICU: Intensive Care Unit; VAP: Ventilator-Associated Pneumonia; SPSS: Statistical Package for Social Science; SAPS: Simplified Acute Physiology Score; SOFA 
Sequential Organ Failure Assessment; MV: Mechanical Ventilation; TA: Tracheal Aspirate; BAL; Bronchoalveolar Lavage; MDRO: Multidrug-Resistant Organisms; ESBL: Extended Spectrum Beta-Lactamase; CI: Confidence Interval.

\section{REFERENCES}

1. Morel J, Casoetto J, Jospé R, Aubert G, Terrana R, Dumont A, et al. De-escalation as part of a global strategy of empiric antibiotherapy management. A retrospective study in a medico-surgical intensive care unit. Crit Care. 2010;14(6):225-31 https://doi.org/10.1186/cc9373 PMid:21167047 PMCid:PMC3219998.

2. Charles MP, Kali A, Easow JM, Joseph NM, Ravishankar M, Srinivasan S, et al. Ventilator-associated pneumonia. Australas Medical J. 2014;7:334-44. https:// doi.org/10.4066/AMJ.2014.2105 PMid:25279009 PMCid:PMC4157153.

3. Gonzalez L, Cravoisy A, Barraud D, Conrad M, Nace L, Lemarié J, et al. Factors influencing the implementation of antibiotic de-escalation and impact of this strategy in critically ill patients. Crit Care. 2013;17(4):140-7. https://doi. org/10.1186/cc12819 PMid:23849321 PMCid:PMC4055984.

4. Khasawneh FA, Karim A, Mahmood T, Ahmed S, Jaffri SF, Mehmood M. Safety and feasibility of antibiotic de-escalation in bacteremic pneumonia. Infect Drug Resist. 2014;7:177-82. https://doi.org/10.2147/IDR.S65928 PMid:25061323 PMCid:PMC4085320.

5. Dellit TH, Owens RC, McGowan JE Jr, Gerding DN, Weinstein RA, Burke JP, et al. Infectious Diseases Society of America and the Society for Healthcare Epidemiology of America guidelines for developing an institutional program to enhance antimicrobial stewardship. Clin Infect Dis. 2007;44(2):159-77. https://doi.org/10.1086/510393 PMid:17173212.

6. Bilal Bin Y, Rozina A, Junaid M, Saima K, Farhan N, Maham T. A Study of Unnecessary Use of Antibiotics at a Tertiary care hospital: urgent need to implement antimicrobial stewardship programs. Journal of Young Pharmacists. 2015;7(4):311-9. https://doi.org/10.5530/jyp.2015.4.5.

7. Bhagawati G, Barkataki D, Hazarika NK. Study on isolates of acute meningitis in a tertiary care centre in Assam. International Journal of Medicine and Public Health. 2014;4(4):446-50. https://doi.org/10.4103/2230-8598.144132.

8. Rani H, Sardana R, Rao P. Voleti P, Rani R. ESBLs producing Enterobacteriaceae in critical care areas - a clinical and cost analysis from a tertiary health care centre. International Journal of Medicine and Public Health. 2012;2(2):49-52. https://doi.org/10.5530/ijmedph.2.2.11, https://doi. org/10.4103/2230-8598.108402.

9. American-Thoracic-Society; Infectious Diseases Society of America. Guidelines for the management of adults with hospital-acquired, ventilatorassociated, and healthcare-associated pneumonia. Am J Respir Crit Care Med. 2005;171:388-416. https://doi.org/10.1164/rccm.200405-644ST PMid:15699079.

10. Eachempati SR, Hydo LJ, Shou J, Barie PS. Does de-escalation of antibiotic therapy for ventilator-associated pneumonia affect the likelihood of recurrent pneumonia or mortality in critically ill surgical patients? J Trauma. 2009;66(5):1343-8. https://doi.org/10.1097/TA.0b013e31819dca4e PMid:19430237.

11. Alvarez-Lerma F, Alvarez B, Luque P, Ruiz F, Dominguez-Roldan JM, Quintana E, et al. Empiric broad-spectrum antibiotic therapy of nosocomial pneumonia in the intensive care unit: a prospective observational study. Crit Care. 2006;10(3):78-88. https://doi.org/10.1186/cc4919 PMid:16704742 PMCid:PMC1550932.
12. Rello J, Vidaur L, Sandiumenge A, Rodríguez A, Gualis B, Boque C, et al. De-escalation therapy in ventilator-associated pneumonia. Crit Care Med. 2004;32:2183-90. https://doi.org/10.1097/01.CCM.0000145997.10438.28 PMid:15640629.

13. Niederman MS. The importance of de-escalating antimicrobial therapy in patients with ventilator-associated pneumonia. Semin Respir Crit Care Med. 2006;27:45-50. https://doi.org/10.1055/s-2006-933673 PMid:16508881.

14. Shelat PR, Gandhi AM, Patel PP. A Study of Drug Utilization Pattern According to Daily Define Dose in Intensive Care Unit (ICU)s at Tertiary Care Teaching Hospital, India. Journal of Young Pharmacists. 2015;7(4):349-58. https://doi.org/10.5530/jyp.2015.4.9.

15. Thomas Z, Bandali F, Sankaranarayanan J, Reardon T, Olsen KM. A Multicenter Evaluation of Prolonged Empiric Antibiotic Therapy in Adult ICUs in the United States. Crit Care Med. 2015;43(12):2527-34. https://doi. org/10.1097/CCM.0000000000001294 PMid:26457751.

16. Rit K, Saha R, Chakraborty B, Majumder U. Ventilator associated pneumonia in a tertiary care hospital in India: Incidence, etiology, risk factors, role of multidrug resistant pathogens. International Journal of Medicine and Public Health. 2014;4:51-6. https://doi.org/10.4103/2230-8598.127125.

17. Leone M, Garcin F, Bouvenot J, Boyadjev I, Visintini P, Albanèse J, et al. Ventilator-associated pneumonia: breaking the vicious circle of antibiotic overuse. Crit Care Med. 2007;35(2):379-85. https://doi.org/10.1097/01. CCM.0000253404.69418.AA PMid:17205011.

18. Garnacho-Montero J, Escoresca-Ortega A, Fernandez-Delgado E. Antibiotic de-escalation in the ICU: how is it best done? Curr Opin Infect Dis. 2015;28(2):193-8. https://doi.org/10.1097/QCO.0000000000000141 PMid:25692272.

19. O'Shea M, Crandon I, Harding H, Donaldson G, Bruce C, Ehikhametalor K. Infections in neurosurgical patients admitted to the intensive care unit at the University Hospital of the West Indies. West Indian Medical J. 2004;53:159-63. PMid:15352744.

20. Josephson SA, Moheet AM, Gropper MA, Nichols AD, Smith WS. Ventilatorassociated pneumonia in a neurologic intensive care unit does not lead to increased mortality. Neurocrit Care. 2010;12(2):155-8. https://doi. org/10.1007/s12028-009-9285-x PMid:19806474.

21. Joung MK, Lee JA, Moon SY, Cheong HS, Joo EJ, Ha YE, et al. Impact of de-escalation therapy on clinical outcomes for intensive care unit-acquired pneumonia. Crit Care. 2011;15(2):79-86. https://doi.org/10.1186/cc10072 PMid:21366903 PMCid:PMC3219332.

22. Kollef $\mathrm{MH}$, Kollef KE. Antibiotic utilization and outcomes for patients with clinically suspected ventilator-associated pneumonia and negative quantitative BAL culture results. Chest. 2005;128(4):2706-13. https://doi. org/10.1378/chest.128.4.2706 PMid:16236946.

23. Giantsou E, Liratzopoulos N, Efraimidou E, Panopoulou M, Alepopoulou E, Kartali-Ktenidou $\mathrm{S}$, et al. De-escalation therapy rates are significantly higher by bronchoalveolar lavage than by tracheal aspirate. Intensive Care Med. 2007;33(9):1533-40. https://doi.org/10.1007/s00134-007-0619-x PMid:17549458.

24. Carlet J, Jarlier V, Harbarth S, Voss A, Goossens H, Pittet D. Ready for a world without antibiotics? The Pensières Antibiotic Resistance Call to Action. Antimicrob Resist Infect Control. 2012;1(1):11-23. https://doi. org/10.1186/2047-2994-1-11 PMid:22958833 PMCid:PMC3436635.

25. Kollef MH, Morrow LE, Niederman MS, Leeper KV, Anzueto A, Benz-Scott L, et al. Clinical characteristics and treatment patterns among patients with ventilator-associated pneumonia. Chest. 2006;129(5):1210-8. https://doi. org/10.1378/chest.129.5.1210 PMid:16685011.

\section{SUMMARY}

- Antibiotic de-escalation practice was low among neurosurgical patients with ventilator-associated pneumonia in the intensive care unit.

- Antibiotic de-escalation therapy is safe among neurosurgical patients with ventilator-associated pneumonia as it was not associated with an excess in mortality. 
- The practice of antibiotic de-escalation should be cultivated among neurosurgical patients with ventilatorassociated pneumonia.

\section{About Authors}

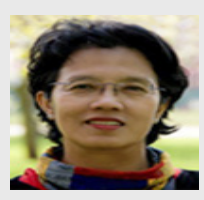

Zoriah Aziz, PhD: Dr Zoriah Aziz is trained in Evidence-based Pharmacotherapy at Aston University in Birmingham, UK. She has been working as a lecturer in the Department of Pharmacy, Faculty of Medicine, University of Malaya, Malaysia since 1998. Prior to that she worked as a pharmacist in the Minisry of Health, Malaysia for 16 years.

Zoriah has a special interest in the assessment of the efficacy and tolerability of herbal medicines using systematic review and meta-analysis methodology. Over the years Zoriah has been very involved in training Malaysian pharmacists in Evidence-based Pharmacotherapy, having co-authored numerous journal articles in the same area.

Zoriah has extensive experience in the area of adverse drug reactions monitoring and pharmacological management of mood disorders, cardiovascular disorders and wounds healing.

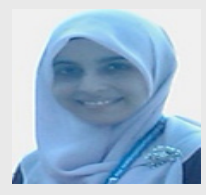

Rahela Ambaras Khan, MPharm: Rahela is working as a Clinical Pharmacist under Ministry of Health Malaysia. She is currently doing her $\mathrm{PhD}$ degree under Faculty of Medicine, University of Malaya Malaysia. She has special interest in critical care field, infectious diseases and antibiotic stewardship programme. She has been involved in publishing protocols and guidelines related to clinical pharmacy in various fields and also participated in publishing the clinical practice guideline for management of tuberculosis and also antibiotic guideline for Ministry of Health Malaysia. She won first place for postgraduate oral presentation in 6th National Pharmacy R\&D Conference in 2010 organised by Pharmaceutical Services Division Ministry of Health Malaysia.

Cite this article: Khan RA, Zoriah Aziz Z. Antibiotic De-escalation Therapy in Neurosurgical Patients with VentilatorAssociated Pneumonia in Intensive Care Unit: A Retrospective Observational Study. Indian J of Pharmaceutical Education and Research. 2017;51(1):144-9. 